\title{
Methodik zur Ermittlung und Validierung von Anforderungen an Studierende (MEVAS)
}

\author{
Anforderungsanalyse \\ für das Fach Wirtschaftswissenschaften
} Benedikt Hell, Claudia Ptok und Heinz Schuler

\begin{abstract}
Zusammenfassung. Die vorliegende Arbeit stellt eine neuartige Methode zur Ermittlung und Validierung von Anforderungen an Studierende (MEVAS) vor. Aufbauend auf den Ergebnissen von Critical Incident Technique-Sitzungen werden in einem zweiten Schritt die ermittelten Einzelanforderungen von Hochschullehrern und Studierenden hinsichtlich der Bedeutsamkeit für ein erfolgreiches Studium und hinsichtlich des Erfüllungsgrads durch derzeit Studierende beurteilt. In einem dritten Schritt werden die Anforderungsdimensionen faktorenanalytisch verdichtet. Die bei den Studierenden festgestellten Erfolgskriterien erlebte Passung im Studium, Studienzufriedenheit, Studienverlaufsdaten und Noten im Vordiplom werden anschließend dazu herangezogen, die ermittelten Anforderungen zu validieren. Die Durchführung der MEVAS im Studiengang Wirtschaftswissenschaften erbringt 15 Anforderungsdimensionen. Die extrahierten Anforderungsdimensionen weisen ein differenzielles Validitätsprofil für verschiedene Studienerfolgskriterien auf. Für das Erfolgskriterium Durchschnittsnote im Vordiplom kann der selbst eingeschätzte individuelle Erfüllungsgrad der Anforderungsdimension Kognitive Leistungsfähigkeit inkrementell Varianz gegenüber dem Prädiktor Abiturdurchschnittsnote aufklären. Die MEVAS kann Grundlage für die Konstruktion von Auswahlverfahren sein und zur Studien- und Karriereberatung herangezogen werden.

Schlüsselwörter: Anforderungsanalyse, Studienerfolg, Studienerfolgsprognose, Auswahl von Studierenden, Studien- und Karriereberatung
\end{abstract}

Method for analyzing the demands of university studies (MEVAS) - requirement analysis for ecconomics and business administration

\begin{abstract}
This study reports a new method for analyzing the demands of university studies (MEVAS). The requirements were identified based on the results of Critical Incident Technique workshops and were then evaluated by university professors and students regarding their importance for successful study and how well they were met by current students. In the third step, a factor analysis of the requirement dimensions was carried out. The criteria for successful study such as perceived fit with the course, satisfaction with the course, study process data, and pre-diploma grades were used to validate the identified requirements. The application of this method to identify and validate study requirements for the subject economics and business administration extracted 15 essential requirement dimensions. The extracted requirement dimensions are characterized by a differential validity profile. MEVAS can provide the basis for the construction of selection methods and can be of use for student and career counselling.

Key words: requirement analysis, academic achievement, academic performance prediction, selection of students, student and career counselling
\end{abstract}

Durch die Novelle des Hochschulrahmengesetzes im Jahre 1998 ist das Interesse an Verfahren zur Auswahl von Studierenden durch die Hochschulen neu erwacht. Für die Entwicklung von Auswahlverfahren, aber auch für die umfassende Information von Schülern und Unentschlossenen und für die Beratung von Studierenden sind sorgfältige Analysen der Anforderungen, die an Studierende eines Studienfachs gestellt werden, unerlässlich.
Darüber hinaus führen die auf der neuen Rechtslage gründende stärkere Autonomie der Hochschulen sowie der zunehmende Wettbewerb zwischen den Hochschulen dazu, dass der gezielten Profilbildung - und damit der Bestimmung und Definition von Anforderungen - in Zukunft eine wachsende Bedeutung zukommt. Eine Sichtung der Literatur zeigt, dass kaum Forschungsarbeit auf dem Gebiet der Bestimmung von Studienanforderungen geleistet 
wurde. Dieses Defizit erschwert die Verständigung über die an Studierende eines Studienfachs zu richtenden Erwartungen seitens der Hochschulen und begünstigt die Aufstellung intuitiv formulierter Anforderungsprofile.

Dieser Beitrag stellt die Methodik zur Ermittlung und Validierung von Anforderungen an Studierende (MEVAS) vor und berichtet ihren Einsatz im Rahmen des Studiengangs Wirtschaftswissenschaften an der Universität Hohenheim. Die Autoren streben an, das Verfahren für weitere Studiengänge durchzuführen. Wir ermuntern interessierte Kollegen ausdrücklich, den vorliegenden Critical Incident-Fragebogen, der bei der MEVAS Anwendung findet, einzusetzen und gegebenenfalls durch weitere studiengangspezifische Items anzureichern ${ }^{1}$.

Die umfangreichsten Erfahrungen in der Durchführung von Arbeits- und Anforderungsanalysen wurden bislang im Rahmen der beruflichen Eignungsdiagnostik gemacht. Begibt man sich auf die Suche nach geeigneten Verfahren für den Kontext der Bestimmung von Anforderungen, stößt man auf Vorgehensweisen, die sich in drei Kategorien einteilen lassen:

Bei der erfahrungsgeleitet-intuitiven Methode setzen sich Experten - also meist Professoren der jeweiligen Fachrichtung - zusammen und brainstormen; sie erstellen also Listen von Eigenschaften (z. B. Rahn et al., 1976). Mit vergleichsweise geringem Aufwand kommen auf diese Art Ergebnisse fragwürdiger Dignität zustande. Es werden beliebige Konstrukte zusammengestellt, die zwar modisch klingen, aber vermutlich weder exhaustiv noch trennscharf sind und deren Validität ungewiss ist.

Aussichtsreicher erscheint die empirisch-korrelative Methode, bei der verfügbare Prädiktoren bei Studienanfängern geprüft und mit dem nachfolgenden Studienerfolg verglichen werden. Die Korrelation zwischen Prädiktor und Kriterium ist in diesem Fall ausschlaggebend für die Anforderungsdefinition. Den Vorteil, dass die Anforderungsdimensionen gleichzeitig validiert sind, erkauft sich der Anforderungsanalytiker teuer, da unklar ist, ob es sich um eine erschöpfende Liste von Prädiktoren handelt - möglicherweise existieren Anforderungsdimensionen, zu deren Erfassung noch keine eignungsdiagnostischen Instrumente zur Verfügung stehen. Zudem handelt es sich um ein langwieriges Verfahren mit einer sehr ungünstigen Kosten-Nutzen-Relation.

Geeigneter als die beiden zuvor genannten Verfahren erscheint die Critical Incident Technique (CIT; Flanagan, 1954). Diese Methode lässt sich ohne weiteres auf den Kontext der Studierendenauswahl übertragen. Ein mögliches Vorgehen bei der Bestimmung der kritischen Ereignisse ist die Durchführung von Workshops mit erfahrenen Studierenden und Hochschullehrern des jeweiligen Fachbereichs. Erfolgreiche Anwendung fand die CIT in

\footnotetext{
${ }^{1}$ Der Fragebogen kann kostenlos beim Erstautor bezogen werden.
}

diesem Bereich z. B. in einer von Schmelzer, Schmelzer, Figler und Brozo (1987) durchgeführten amerikanischen Untersuchung, deren Ziel die Feststellung der Ursachen für Erfolg oder Misserfolg im Studium war. Das Verfahren kann ökonomisch angewendet werden, ist aber in seiner Qualität in hohem Maße von der Kompetenz und Erfahrung des durchführenden Arbeitsanalytikers abhängig. Eine empirische Prüfung der definierten Anforderungsdimensionen findet nicht statt.

\section{Ziel der Untersuchung}

Im Zuge der vorliegenden Untersuchung wird ein standardisiertes Vorgehen zur Bestimmung von Studienanforderungen vorgestellt. Es handelt sich um eine Fortentwicklung des Fragebogens Bedeutsamkeit und Erfüllungsgrad von Anforderungen (Schuler, 1996, 2002).

Mit der Methode zur Ermittlung und Validierung von Anforderungen an Studierende ist es möglich, Anforderungen für einzelne Studiengänge zu bestimmen und deren Aussagegehalt zugleich an von Studierenden für die eigene Person eingeschätzten Erfolgskriterien zu prüfen. Durch ein standardisiertes Vorgehen wird sowohl eine hohe Qualität als auch eine Vergleichbarkeit von Ergebnissen verschiedener Anforderungsanalysen gewährleistet.

Die MEVAS besteht aus folgenden Arbeitsschritten:

1. Durchführung von Critical Incident-Workshops mit Hochschullehrern und erfahrenen Studierenden.

2. Sichtung und redaktionelle Bearbeitung der gewonnenen Itemliste.

3. Abgleich und gegebenenfalls Anreicherung der Itemliste mit Items aus vorhandenen Untersuchungen.

4. Empirische Prüfung der Items: Bestimmung des Grads, in dem die Anforderungen erfüllt sind; Feststellung der Bedeutsamkeit sowohl durch Studierende als auch durch Hochschullehrer.

5. Ermittlung von Studienerfolgskriterien bei den Studierenden.

6. Gegebenenfalls Reduktion der Items durch Elimination von nicht bedeutsamen Items (Einschätzung durch Hochschullehrer).

7. Bestimmung der Anforderungsdimensionen durch Strukturanalysen des Erfüllungsgrads (Selbsteinschätzung der Studierenden).

8. Gegenüberstellung der Bedeutsamkeits- und Erfüllungsgradeinschätzungen.

9. Validierung der Anforderungsdimensionen (Selbsteinschätzung Erfüllungsgrad durch die Studierenden) an den Erfolgskriterien.

Die erarbeitete Methode verbindet die Vorteile einer spezifischen Itemsammlung in einer qualitativen Phase mit einer nachfolgenden empirischen Prüfung. Im Kon- 
text der Bestimmung von Anforderungen an Studierende ist es möglich, das von Schuler (1996) vorgestellte Vorgehen zu erweitern. So ist es hier möglich, größere Gruppen von Studierenden zu befragen. Dies erlaubt den Einsatz von multivariaten Verfahren und führt zu stabilen und interpretierbaren faktorenanalytischen Lösungen. Der Grad, in dem die Anforderungen erfüllt sind, kann als Fremdurteil bei Professoren und als Selbsturteil bei Studierenden erhoben werden (Instruktion Professoren: „Inwieweit erfüllen die Studierenden Anforderung X“; Instruktion Studierende: „Inwieweit erfüllen Sie persönlich die Anforderung $X^{\text {" }}$ ). Im vorliegenden Kontext ist es möglich, die Selbsteinschätzungen der Studenten einer Faktorenanalyse zu unterziehen. Hierbei werden - nicht wie bei faktorenanalytischen Untersuchungen von Bedeutsamkeitseinschätzungen, die in der Regel einen varianzstarken ersten Bedeutsamkeitsfaktor erbringen vollständig interpretierbare Lösungen erreicht.

Bei der Analyse von Studienanforderungen lassen sich Erfolgskriterien wie Studiennoten bei den Studierenden ökonomisch und zudem valide ermitteln. Die Selbsteinschätzung der Studierenden, inwieweit die Anforderungen erfüllt sind, kann mit den erhobenen Kriteriendaten verglichen werden. Hierdurch wird eine Validierung der Anforderungsdimensionen gewährleistet.

Es gibt einen gemeinsamen Kern an Anforderungen, die an Studierende verschiedener Studienfächer gestellt werden. Folglich kann ein Teil der Items des hier vorgestellten Anforderungsfragebogens bei weiteren zu analysierenden Studiengängen eingesetzt werden.

\section{Anforderungsanalyse für das Fach Wirtschaftswissenschaften}

\section{Methode}

Im Rahmen der durchgeführten Anforderungsanalyse wurden zwei Zielgruppen - Studierende im Grund$(N=113)$ und Hauptstudium $(N=89)$ sowie Hochschullehrer $(N=27)$ - der wirtschaftswissenschaftlichen Fakultät der Universität Hohenheim befragt (die Gruppe der Studierenden wird in Grund- und Hauptstudium differenziert, da in Abhängigkeit von der Studienerfahrung unterschiedliche Bewertungen möglich sein könnten). Die durchschnittliche Dauer der Lehr- und Forschungstätigkeiten der befragten Hochschullehrer lag bei 20.11 Jahren, mit einem minimalen Wert in Höhe von 3 und einem maximalen Wert in Höhe von 38 Lehr- und Forschungsjahren.

In einem ersten Schritt wurden drei Critical IncidentWorkshops mit insgesamt 8 weiblichen und 8 männlichen erfahrenen Studierenden durchgeführt. Die Bestimmung der kritischen Ereignisse erfolgte innerhalb drei jeweils einstündiger Workshops, an denen im Hauptstudium studierende Wirtschaftswissenschaftsstudenten der Universität Hohenheim teilnahmen. Die Workshops liefen in vier Schritten ab. Nach einer im ersten Teilschritt durchgeführten knappen Darstellung der Thematik und allgemeinen Verfahrenseinführung erhielten die Teilnehmer die Instruktion, das Studierverhalten eines Kommilitonen bzw. das eigene Studierverhalten zu beschreiben, das eine besonders effektive oder ineffektive Arbeitsweise veranschaulicht. Im zweiten Schritt erhielten die Teilnehmer die Aufgabe, innerhalb von 20 Minuten kritische Ereignisse auf dafür vorgefertigte Formulare zu notieren, die im anschließenden dritten Schritt an eine für alle Teilnehmer ersichtliche Tafel geheftet und mündlich von dem jeweiligen Teilnehmer erläutert wurden. Der vierte und letzte Teilschritt der Workshops bestand in der Diskussion zwischen den Teilnehmern und der Erfassung daraus resultierender ergänzender kritischer Ereignisse.

Die gewonnenen 248 kritischen Verhaltensweisen wurden redaktionell bearbeitet, Redundanzen wurden eliminiert und es wurden Items von den Autoren und aus einschlägigen Publikationen ergänzt (Heldmann, 1984; Konegen-Grenier, 2002; Trost \& Bickel, 1979). In der Endfassung enthält der Fragebogen 167 Items.

Es wurden unterschiedliche Varianten für die studentischen Stichproben und für die Hochschullehrer zusammengestellt. Die Versionen unterscheiden sich hinsichtlich der Perspektive bei der Einschätzung des Erfüllungsgrads der Anforderungen: Die Einschätzung des Erfüllungsgrads durch die Studierenden wird als Selbsteinschätzung, die durch die Hochschullehrer in Form einer Fremdeinschätzung erhoben. Zudem wurde die Studierendenversion durch einen Kriterienbogen ergänzt, der eine Validierung der ermittelten Anforderungen erlaubt. Als Studienerfolgsindikatoren werden die erlebte Passung im Studium, die Studienzufriedenheit, verschiedene Studienverlaufsdaten sowie Noten im Vordiplom herangezogen. Zur Messung der erlebten Passung im Studium kam eine von Rolfs und Schuler (2002 a, 2002 b) konstruierte Skala in modifizierter Form zum Einsatz. Zur Erhebung der Studienzufriedenheit fanden Ausschnitte eines von Westermann, Heise, Spies und Trautwein (1996) erarbeiteten Fragebogens Verwendung. Er wurde ergänzt durch Items aus Trost und Bickel (1979) sowie Lewin und Schacher (1982). Die Messung der Studienverlaufsdaten orientiert sich an einer von Kazemzadeh und Schaeper (1984) durchgeführten Studie.

\section{Ergebnisse}

\section{Bestimmung der Anforderungsdimensionen}

Zunächst wurde der Itempool auf diejenigen Items reduziert, die von Seiten der Hochschullehrer im Hinblick auf den Studienerfolg im Mittel mindestens als hoch bedeut- 
sam (Bedeutsamkeit $\geq 3$ ) eingeschätzt wurden. Anschließend wurden die verbleibenden 133 Items einer Faktorenanalyse unterzogen. Grundlage hierfür waren die durch die Studierenden vorgenommenen Selbsteinschätzungen des Erfüllungsgrads. Als Extraktionsmethode kam die Hauptkomponentenanalyse, als Extraktionskriterium die Hornsche Parallelanalyse (Horn, 1965) zur Anwendung. Nach Maßgabe des Ergebnisses der Hornschen Parallelanalyse wurden 11 Faktoren extrahiert, die zusammen $42.4 \%$ der Varianz der 133 Variablen aufklären. Der erste Faktor leistet eine Varianzaufklärung in Höhe von $8.5 \%$. Die Varianzaufklärung der restlichen 10 extrahierten Faktoren lag jeweils zwischen $4.1 \%$ und $2.2 \%$. Bei vier Faktoren zeigte sich sehr deutlich, dass sie inhaltlich verschiedene Bereiche vereinten. Sie wurden auf Basis inhaltlicher Überlegungen ausdifferenziert (nachfolgend gekennzeichnet durch den Zusatz $a / b$ ), wodurch sich die Anzahl der Faktoren auf 15 erhöhte. Drei Items konnten nicht eindeutig einem Faktor zugeordnet werden und wurden folglich nicht weiter berücksichtigt. Ein zusammenfassender Überblick der Ergebnisse findet sich in Tabelle 1. Die entsprechenden Kennwerte der internen Konsistenz liegen zwischen $\alpha=.45$ und $\alpha=.90$. Die Dimension Flexibilität weist mit einem Cronbachs $\alpha$ in Höhe von .45 bei einer Gesamtzahl von drei Items eine unzureichende Homogenität auf und wird daher bei den weiteren Analysen nicht berücksichtigt.

\section{Vergleich der Einschätzungen zwischen den Untersuchungsgruppen}

Der Vergleich der von den drei Untersuchungsgruppen (Studierende im Grund- und Hauptstudium, Hochschullehrer) jeweils vorgenommenen Einschätzungen des Erfüllungsgrads zeigt, dass teilweise unterschiedliche Sichtweisen vorherrschen: Bis auf die Dimensionen 5 und $7 \mathrm{~b}$ differieren die gemittelten Urteile signifikant (einfaktorielle Varianzanalysen, Faktor: Untersuchungsgruppe). Die gefundenen Unterschiede sind besonders interessant, da hier Selbsturteilen Fremdurteile gegenüberstehen (vgl. Abbildung 1).

Größere Defizite erkennen die Professoren bei den Studierenden in den Bereichen Kognitive Leistungsfähigkeit, Gewissenhaftigkeit sowie Identifikation mit dem Studium und sorgfältige Studienfachwahl. Weiterhin sind sie mit dem Literaturstudium der Studierenden besonders unzufrieden (Dimension Lesefreude und proaktives Literaturstudium).

\section{Validierung der Anforderungsdimensionen}

Die bei den Studierenden erfragten Studienerfolgskriterien wurden mittels Item- und Skalenanalysen geprüft. Eine ausreichende Homogenität ist bei allen Kriterien gegeben (Cronbachs $\alpha$ zwischen .79 bzw. .87). Einzig das Kriterium Studienverlaufsdaten erwies sich als hete- rogen. Erst eine Zusammenfassung der Fragen zu einem dichotomen Studienverlaufsindex führte zu einem psychometrisch hinreichenden Kriterium. Bei der Indexgenerierung wurde allen Antwortalternativen, die auf das Vorliegen einer Besonderheit hinweisen (Studienunterbrechung, Erwägen eines Studienabbruchs in der Vergangenheit, Absicht eines Studienabbruchs, in der Vergangenheit durchgeführte Studiengangwechsel, in der Vergangenheit ernsthaft erwogene Studiengangwechsel, zukünftig beabsichtigte Studiengangwechsel) die Ausprägung 1 , allen übrigen eine 0 zugewiesen.

Die Validität der Anforderungsdimensionen wird überprüft, indem die durch die Studierenden selbst eingeschätzten Erfüllungsgrade der Dimensionen zu den genannten Kriterien in Beziehung gesetzt werden. Hierzu werden für die erhobenen Studienerfolgskriterien erlebte Passung im Studium, Studienzufriedenheit, Studienverlaufsdaten sowie Noten im Vordiplom sequenziell zwei multiple Regressionen berechnet. Eine im ersten Schritt durchgeführte multiple Regression verfolgt das Ziel, diejenigen Anforderungsdimensionen aufzudecken, die einen signifikanten Erklärungsbeitrag hinsichtlich des jeweiligen Kriteriums erbringen (Aufnahmemethode: schrittweise, rückwärts). Ihr kommt eine Art Filterfunktion zu: Dimensionen, die in der ersten Regression negative Regressionskoeffizienten erhalten, werden bei der zweiten Analyse ausgeschlossen. Das Signifikanzniveau wird auf einem liberalen Niveau in Höhe von $p \leq .10$ angesetzt, um zu verhindern, dass bei diesem Analyseschritt Anforderungsdimensionen eliminiert werden, die einen Erklärungsbeitrag leisten.

Eine Ableitung der Stärke des Erklärungsbeitrags der herausgefilterten Anforderungsdimensionen erfolgt mittels einer zweiten multiplen Regression. In diesem Schritt wurden zunächst alle Anforderungsdimensionen aufgenommen, die sich bei der im ersten Schritt durchgeführten multiplen Regression als signifikant und von der Richtung des Zusammenhangs aus betrachtet als inhaltlich sinnvoll erwiesen haben. Schrittweise werden dann diejenigen Anforderungsdimensionen eliminiert, die keinen bedeutsamen Erklärungsbeitrag leisten (Methode: schrittweise, rückwärts). Das Ergebnis dieses zweiten Schritts ist in Tabelle 2 aufgeführt.

Es ist leicht zu erkennen, dass die Anforderungsdimensionen einen differenziellen Bezug zu den Kriterien aufweisen. Für das von vielen Autoren als am wichtigsten angesehene Kriterium Studiennoten ist einzig die Dimension Kognitive Leistungsfähigkeit valide. Eine neben der Kognitiven Leistungsfähigkeit besonders bedeutende Anforderung stellt die Identifikation mit dem Studium und sorgfältige Studienfachwahl dar. Sie ist für die Kriterien Erlebte Passung und Studienzufriedenheit hoch valide und kovariiert darüber hinaus systematisch mit den Studienverlaufsdaten. 
Tabelle 1. Übersicht über die extrahierten Anforderungsdimensionen

\begin{tabular}{|c|c|c|c|c|}
\hline \multicolumn{2}{|c|}{ Dimensionsbezeichnung } & \multirow{2}{*}{$\begin{array}{l}\text { Beispielitems } \\
\text { - Urteilsvermögen } \\
\text { - analytisch denken }\end{array}$} & \multirow{2}{*}{$\begin{array}{l}\begin{array}{l}\text { Item- } \\
\text { anzahl }\end{array} \\
18\end{array}$} & \multirow{2}{*}{$\begin{array}{l}\text { Cron- } \\
\text { bachs } \alpha \\
90\end{array}$} \\
\hline D 1 & Kognitive Leistungsfähigkeit & & & \\
\hline D 2 & Lern- und Arbeitstechniken & $\begin{array}{l}\text { - sich alte Klausuren als Lerngrundlage beschaffen } \\
\text { - eigene Zusammenfassungen des Lernstoffs erstellen }\end{array}$ & 16 & .81 \\
\hline D $3 \mathrm{a}$ & Gewissenhaftigkeit & $\begin{array}{l}\text { - zuverlässig sein } \\
\text { - Genauigkeit und Sorgfalt }\end{array}$ & 5 & .69 \\
\hline D $3 b$ & $\begin{array}{l}\text { Identifikation mit dem } \\
\text { Studium und sorgfältige } \\
\text { Studienfachwahl }\end{array}$ & $\begin{array}{l}\text { - inhaltliches Interesse } \\
\text { - sich über den Studiengang und seine Anforderungen } \\
\text { informieren }\end{array}$ & 10 & .77 \\
\hline D 4 & $\begin{array}{l}\text { Lesefreude und proaktives } \\
\text { Literaturstudium }\end{array}$ & $\begin{array}{l}\text { - Fachzeitschriften lesen } \\
\text { - das Angebot der Bibliothek und der Online-Medien } \\
\text { nutzen }\end{array}$ & 10 & .77 \\
\hline D 5 & $\begin{array}{l}\text { Berufseinstiegserleichternde } \\
\text { Zusatzqualifikationen }\end{array}$ & $\begin{array}{l}\text { - Praxisbezug durch Praktika herstellen } \\
\text { - Sprachkurse belegen }\end{array}$ & 8 & .86 \\
\hline D $6 \mathrm{a}$ & $\begin{array}{l}\text { Effektive und zielgerichtete } \\
\text { Zeit-, Lern- und Studien- } \\
\text { organisation }\end{array}$ & $\begin{array}{l}\text { - sich bei der Klausurvorbereitung einen Zeitplan } \\
\text { aufstellen und einhalten } \\
\text { - sich konkrete Lernziele pro Semester setzen }\end{array}$ & 9 & .77 \\
\hline D $6 \mathrm{~b}$ & $\begin{array}{l}\text { Leistungsmotivation } \\
\text { und Belastbarkeit }\end{array}$ & $\begin{array}{l}\text { - Eigeninitiative } \\
\text { - Leistungsmotivation }\end{array}$ & 12 & .83 \\
\hline D $7 \mathrm{a}$ & $\begin{array}{l}\text { Techniken zur Anfertigung } \\
\text { wissenschaftlicher Arbeiten }\end{array}$ & $\begin{array}{l}\text { - gute EDV-Kenntnisse } \\
\text { - sich mit der Vorgehensweise bei der Literaturrecherche } \\
\text { für Seminararbeiten/Diplomarbeit vertraut machen }\end{array}$ & 8 & .73 \\
\hline $\mathrm{D} 7 \mathrm{~b}$ & $\begin{array}{l}\text { Strategische } \\
\text { Prüfungsvorbereitung }\end{array}$ & $\begin{array}{l}\text { - sich bei Prüfungen auf den Professor einstellen } \\
\text { - für mündliche Prüfungen lieber breit als tief lernen }\end{array}$ & 3 & .53 \\
\hline D $8 \mathrm{a}$ & $\begin{array}{l}\text { Proaktives } \\
\text { Informationsverhalten }\end{array}$ & $\begin{array}{l}\text { - Fragen zur Vertiefung beim betreffenden Lehrstuhl klären } \\
\text { - Internet als Hilfsmittel zur Informationsgewinnung nutzen }\end{array}$ & 8 & .64 \\
\hline $\mathrm{D} 8 \mathrm{~b}$ & Flexibilität & $\begin{array}{l}\text { - in der Studienplanung flexibel sein } \\
\text { - Flexibilität }\end{array}$ & 3 & .45 \\
\hline D 9 & Studieren nach Studienplan & $\begin{array}{l}\text { - sich an den vorgegebenen Studienplan halten } \\
\text { - regelmäßig Vorlesungen besuchen }\end{array}$ & 7 & .74 \\
\hline D 10 & Gelassenheit & $\begin{array}{l}\text { - in Prüfungssituationen ruhig und gelassen bleiben } \\
\text { - sich durch schlechte Prüfungsergebnisse } \\
\text { nicht demotivieren lassen }\end{array}$ & 9 & .78 \\
\hline D 11 & $\begin{array}{l}\text { Überlegte } \\
\text { Vertiefungsfachwahl }\end{array}$ & $\begin{array}{l}\text { - Vertiefungsfachwahl von den eigenen Interessen } \\
\text { abhängig machen } \\
\text { - Vertiefungsfächer aufeinander abstimmen }\end{array}$ & 4 & .54 \\
\hline
\end{tabular}




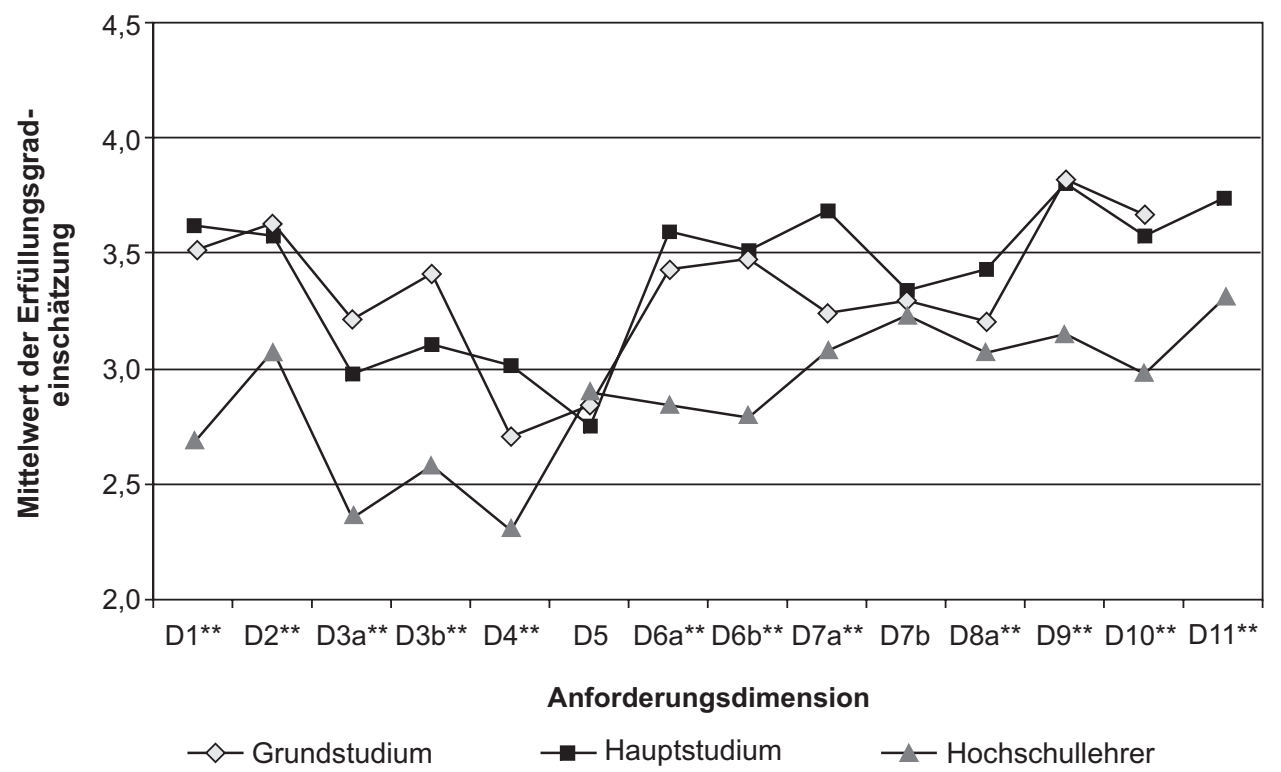

Anmerkungen. 1 = sehr geringer, 5 = sehr hoher Erfüllungsgrad; für die Dimension 11 wurde ein t-Test für unabhängige Stichproben berechnet (wurde nicht von Studierenden des Grundstudiums bearbeitet), die restlichen Dimensionen wurden mittels einfaktorieller ANOVAs analysiert; $* * p \leq .01$.

Abbildung 1. Mittelwertvergleich der Erfüllungsgradeinschätzung.

\section{Diskussion}

\section{Anforderungsanalyse für das Fach Wirtschaftswissenschaften}

Die herausgearbeiteten Anforderungsdimensionen für das Fach Wirtschaftswissenschaften können zur Konstruktion von Auswahlverfahren für dieses Studienfach genutzt werden. Je nach Anforderungsdimension bieten sich unterschiedliche eignungsdiagnostische Verfahren an. Die Dimension Kognitive Leistungsfähigkeit kann zum Teil durch Abiturnoten abgebildet werden. Ergänzend könnten Leistungstests eingesetzt werden, deren inkrementelle Validität belegt ist (Trost, 1998). Für eine Operationalisierung der Dimensionen Identifikation mit dem Studium und sorgfältige Studienfachwahl sowie Leistungsmotivation und Belastbarkeit bieten sich sowohl interaktive Verfahren (z. B. Interviews) als auch standardisierte schriftliche Verfahren wie biografische Fragebogen oder die Analyse von Bewerbungsunterlagen an. Es erscheint auch aussichtsreich, diese Anforderungsdimensionen über Selbsteinschätzungen abzubilden. Interessen-, Persönlichkeits- und Motivationstests können über das Internet Studieninteressierten zur Verfügung gestellt werden, um eine Selbstselektion der Bewerber zu ermöglichen. Zur Bestimmung, welche Anforderungen zur Eignungsdiagnose genutzt werden, sollten nicht nur die berechneten Zusammenhänge der Dimensionen mit den Erfolgskriterien geprüft werden. Eine weitere Entscheidungsgrundlage bieten die von den Dozenten abgegebenen Bedeutsamkeitseinschätzungen der Anforderungen.
Sie können sowohl in ihrer absoluten Höhe als auch im Vergleich zu den Erfüllungsgradeinschätzungen analysiert werden. Ein Vergleich der eingestuften Bedeutsamkeiten mit den Erfüllungsgraden zeigt eine von den befragten Hochschullehrern wahrgenommene deutliche und unerwünschte Diskrepanz zwischen dem Erfüllungsgrad der Dimension Gewissenhaftigkeit sowie der Dimension Lesefreude und proaktives Literaturstudium und der ihnen zugesprochenen Bedeutsamkeit. Die Hochschullehrer sind offenbar der Ansicht, dass diese für ein erfolgreiches Studium aus ihrer Sicht wichtigen Aspekte von den Studierenden in unzureichendem Maße erfüllt werden. Die genannten Merkmale sollten bei zukünftigen Auswahlentscheidungen demnach ein größeres Gewicht erhalten. Beide genannten Aspekte könnten durch Interviews oder durch biografische Fragebogen abgedeckt werden.

Auch Coaching-Maßnahmen wie Studien- oder Karriereberatung sollten ihren Ausgangspunkt in fundierten Anforderungsanalysen haben. Die Bedeutsamkeit der Anforderungsdimension Identifikation mit dem Studium und sorgfältige Studienfachwahl ist in diesem Zusammenhang besonders beachtenswert und ein Hinweis darauf, dass sich Investitionen in die Studienberatung von Studieninteressierten rentieren könnten. Eine realistische Tätigkeits- und Studieninformation (vgl. Wanous, 1980) sollte zu einer geringeren Abbruchquote und einer größeren Studienzufriedenheit führen. Aber auch die Validität der Dimension Studieren nach Studienplan weist den Weg in Richtung einer intensiveren Betreuung von Studierenden durch Informations- und Coaching-Angebote. 
Tabelle 2. Kriterienbezug der Anforderungsdimensionen

\begin{tabular}{|c|c|c|c|}
\hline \multirow[t]{2}{*}{ Kriterium } & \multicolumn{3}{|l|}{ Prädiktormodell } \\
\hline & Prädiktor & Beta & $\begin{array}{l}\text { korrigiertes } R^{2} \\
\text { (Sign.) }\end{array}$ \\
\hline \multirow[t]{2}{*}{ Erlebte Passung im Studium } & $\begin{array}{l}\text { Identifikation mit dem Studium und sorgfältige } \\
\text { Studienfachwahl (D } 3 \text { b) }\end{array}$ & $.43^{* *}$ & $.33^{* *}$ \\
\hline & Leistungsmotivation und Belastbarkeit (D 6 b) & $.22 * *$ & \\
\hline \multirow[t]{2}{*}{ Studienzufriedenheit } & $\begin{array}{l}\text { Identifikation mit dem Studium und sorgfältige } \\
\text { Studienfachwahl (D } 3 \text { b) }\end{array}$ & $.50 * *$ & $.42^{* *}$ \\
\hline & Leistungsmotivation und Belastbarkeit (D 6 b) & $.23 * *$ & \\
\hline \multirow[t]{2}{*}{ Studienverlaufsdaten } & $\begin{array}{l}\text { Identifikation mit dem Studium und sorgfältige } \\
\text { Studienfachwahl (D } 3 \text { b) }\end{array}$ & .12 n.s. & $.06^{* *}$ \\
\hline & Studieren nach Studienplan (D 9) & $.20 * *$ & \\
\hline Noten im Vordiplom & Kognitive Leistungsfähigkeit (D 1) & $.42 * *$ & $.17^{* *}$ \\
\hline
\end{tabular}

Anmerkungen. ${ }^{* *} p \leq .01$

\section{Nutzen und Anwendungsfelder der MEVAS}

Mit dem beschriebenen Vorgehen ist es möglich, Anforderungen, die an Studierende verschiedener Studienrichtungen gestellt werden, systematisch und in einem standardisierten Prozedere festzulegen. Wir möchten dazu ermuntern, die Methode bei anderen Studiengängen einzusetzen. $\mathrm{Zu}$ diesem Zweck können die vorhandenen Items verwendet und durch weitere studiengangspezifische Items ergänzt werden. Durch einen Vergleich der Ergebnisse wird es möglich sein, Studienanforderungen, die an alle Studierende gestellt werden, von studienfachspezifischen Anforderungen zu trennen. Durch die Einbeziehung von Unternehmen, die als zukünftige Arbeitgeber der Hochschulabgänger die Endverbraucher des Bildungsmarkts darstellen, könnte bei Nachfolgestudien ein weiterer interessanter Blickwinkel zu den bereits berücksichtigten hinzutreten.

Bei der Festlegung des Stichprobenumfangs für Schritt 4 der MEVAS ist die Anzahl der Items als maßgebliches Entscheidungskriterium heranzuziehen (vgl. MacCallum, Widaman, Zhang \& Hong, 1999). Die dieser Untersuchung zugrunde liegende Relation von Items $\mathrm{zu}$ Versuchpersonen ist als Untergrenze anzusehen.

Die Bestimmung von multiplen Validierungskriterien hat sich als sinnvoll erwiesen, da eine differenzielle Validität der Anforderungsdimensionen für die verschiedenen Kriterien nachgewiesen werden konnte. Gleichwohl muss an dieser Stelle auf die Grenzen der Trennbarkeit von Anforderungsdimension und Kriterium hingewiesen werden. Das Erhebungsdesign ist nicht unproblematisch: Die anforderungsanalytisch gewonnenen Indikatoren sind keine Prädiktoren im Wortsinne. Vielmehr wurden sie im Fall des Kriteriums Vordiplomnote zeitlich nach dem Kriterium erhoben. Die Kriterienleistungen wirken sich daher möglicherweise auf die Selbsteinschätzungen der Erfüllungsgrade der Anforderungsdimensionen aus. Diejenigen Studierenden, die gute Noten im Vordiplom erzielt haben, schätzen ihre kognitive Leistungsfähigkeit höher ein als diejenigen, die schlechte Noten erzielt haben. Dennoch halten wir die berechneten Validitäten für aussagekräftig. An ein Praxisprojekt wie eine Anforderungsanalyse können nicht die gleichen Qualitätskriterien wie an eine experimentelle Studie oder an eine Testentwicklung gestellt werden. In jedem Fall sollten die Schwierigkeiten, die mit der konkurrenten Kriterienerhebung verbunden sind, nicht außer Acht gelassen werden. Soweit möglich und rechtlich zulässig, wäre es von Vorteil, Leistungsdaten aus den Prüfungsämtern als ergänzende Daten heranzuziehen.

Schließlich besteht die Möglichkeit, die hier geschilderte Methodik wieder in jenem Bereich zur Anwendung zu bringen, dem ihre Vorform entstammt: Das Analyseverfahren Bedeutsamkeit und Erfüllungsgrad von Anforderungen (Schuler, 1996, 2002) wurde in einer Vielzahl von Berufsfeldern und Tätigkeitsniveaus als Basis personalpsychologischer Maßnahmen eingesetzt. Spezifisch bieten sich die Bedeutsamkeitswerte als Hinweise für die Personalauswahl und die Erfüllungsgradwerte als Richtwerte für Maßnahmen der Personalentwicklung an. Die hier geschilderten methodischen Weiterentwicklungen dieses Verfahrens können auch der berufsbezogenen Anforderungsanalyse und damit personalpsychologischen Zwecken zugute kommen. 


\section{Literatur}

Flanagan, J. C. (1954). The critical incident technique. Psychological Bulletin, 51, 327-358.

Heldmann, W. (1984). Studierfähigkeit - Ergebnisse einer Umfrage. Göttingen: Schwartz.

Horn, J. L. (1965). A rationale and test for the number of factors in factor analysis. Psychometrica, 30, 179-185.

Kazemzadeh, F. \& Schaeper, H. (1984). Wer findet sich im Studium zurecht? Ergebnisse einer Untersuchung von Studenten in der Eingangsphase des Studiums (Hochschulplanung, Bd. 52). Hannover: HIS GmbH.

Konegen-Grenier, C. (2002). Studierfähigkeit und Hochschulzugang. Köln: Deutscher Instituts-Verlag.

Lewin, K. \& Schacher, M. (1982). Studienberechtigte '78 - Studienaufnahme, Studienfinanzierung, Zufriedenheit: Bestandsaufnahme zwei Jahre nach Erwerb der Hochschulreife und Vergleich mit Studienberechtigten '76 (Hochschulplanung, Bd. 42). Hannover: HIS GmbH.

MacCallum, R. C., Widaman, K. F., Zhang, S. \& Hong, S. (1999). Sample size in factor analysis. Psychological Methods, 4, 84-99.

Rahn, H. et al. (1976). Modellversuch „Studienfeldbezogene Tests “. Erster Arbeitsbericht: 1. Oktober 1975 bis 31. Juli 1976. Bonn: Institut für Test- und Begabungsforschung.

Rolfs, H. \& Schuler, H. (2002 a). Berufliche Interessenkongruenz und das Erleben im Studium. Zeitschrift für Arbeitsund Organisationspsychologie, 46, 137-149.

Rolfs, H. \& Schuler, H. (2002 b). Lernstrategien im Studium als Korrelat beruflicher Interessenkongruenz. Psychologie in Erziehung und Unterricht, 49, 250-262.
Schmelzer, R. V., Schmelzer, C. D., Figler, R. A. \& Brozo, W. G. (1987). Using the critical incident technique to determine reasons for success and failure of university students. Journal of College Student Personnel, 28, 261-266.

Schuler, H. (1996). Psychologische Personalauswahl. Göttingen: Hogrefe.

Schuler, H. (2002). Das Einstellungsinterview. Göttingen: Hogrefe.

Trost, G. (Hrsg.). (1998). Evaluation des Tests für medizinische Studiengänge (TMS): Synopse der Ergebnisse. Bonn: Institut für Test- und Begabungsforschung.

Trost, G. \& Bickel, H. (1979). Studierfähigkeit und Studienerfolg. München: Minerva.

Wanous, J. P. (1980). Organizational entry: Recruitment, selection, and socialization of newcomers. Reading, MA: Addison-Wesley.

Westermann, R., Heise, E., Spies, K. \& Trautwein, U. (1996). Identifikation und Erfassung von Komponenten der Studienzufriedenheit. Psychologie in Erziehung und Unterricht, 43, 1-22.

Eingegangen: 20.06.2005

Revision eingegangen: 02.11.2005

Dr. Benedikt Hell

Lehrstuhl für Psychologie (540F)

Universität Hohenheim

70593 Stuttgart

E-Mail: hell@uni-hohenheim.de 